\section{Presidential address 2016}

\section{Towards Ending AIDS in Sri Lanka}

\section{Dr Ajith Karawita}

Presidential address at the inaugural session of the Sri Lanka College of Sexual Health and HIV Medicine (Sri Lanka CoSHH), 13-15 October 2016, Kingsbury, Colombo.

Chief Gust Dr Palitha Mahipala

Guest of honour Dr David Barlow

Distinguished invitees from abroad

Past presidents

Fellows of the college

Members of the council of the Sri Lanka

College of Sexual health and HIV Medicine

Members of the college

My teachers, friends, ladies and gentlemen,

The theme of the $21^{\text {st }}$ Annual Scientific Sessions and today's my keynote address is on

"Towards ending AIDS in Sri Lanka"

My presentation include

Introduction

Global scenario in ending AIDS

HIV epidemic in Sri Lanka

Ending AIDS in Sri Lanka

\section{Introduction}

As you know, the Human Immunodeficiency Virus (HIV) damages the smooth functioning of your immune system leading to immune deficiency and dysregulation.

HIV targets important cells in the immune system. The central immune defect is the infection of CD4+ lymphocytes leading to the destruction and depletion of cells with increase in HIV viraemia and emergence of opportunistic diseases.

Clinical presentations, CD4+ lymphocyte count and HIV viral load are the main parameters to understand the level of the progression of HIV disease.

Natural history of HIV mainly consists of asymptomatic illness followed by period of opportunistic infections and opportunistic malignancies. After many revisions and updates, WHO has recommended clinical stage-based approach for the evaluation of clinical disease in HIV.

HIV acquisition followed by a viral fever like illness called seroconversion illness. Then there is a long latency about 8-12 years called clinical stage I which is followed by symptomatic diseases of clinical stage II, III, and IV as depicted in the slide

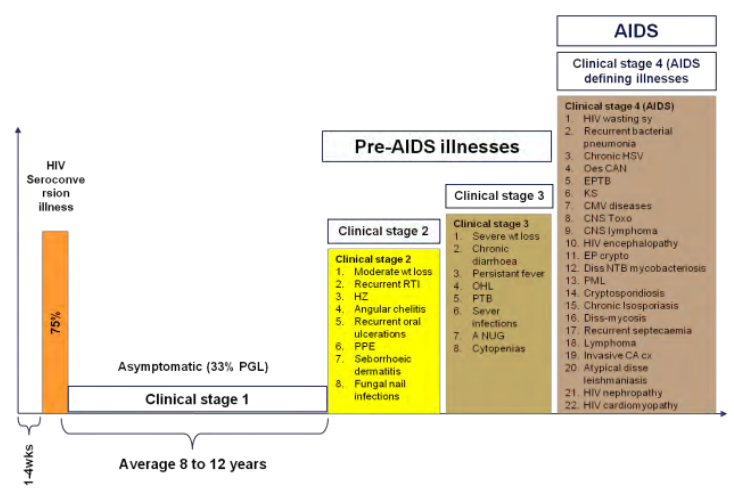

Clinical stage I is generally the asymptomatic stage, clinical stage II and III are pre-AIDS illnesses. If a patient is having stage IV illnesses, he or she is categorized as having AIDS.

Fortunately in HIV, those with clinical stage II, III and IV illnesses can be reversed by adequate suppression of viraemia. Therefore, antiretrovirals and management of opportunistic diseases can keep infected persons at stage $\mid$ increasing the life expectancy and quality of life till complete cure is available.

Therefore, you can end or stop the progression of the disease, to its advanced stage called AIDS. In other words, you can end AIDS theoretically and scientifically by own immunity supported by antiretroviral drugs. 
However, the problem is that, whether we could deliver this science to our community, scale enough to end AIDS in human population. Therefore, we need to put lots of effort for programme and implementation science more and more to reach every person concerned.

The United Nations Political Declaration on ending AIDS done on $8^{\text {th }}$ June 2016 sets world on the fast track to end the epidemic by 2030 . Countries have agreed, to a historic and urgent agenda to accelerate efforts towards ending the AIDS epidemic by 2030 .

The ambitious 90-90-90 triple target is the treatment target towards ending AIDS.

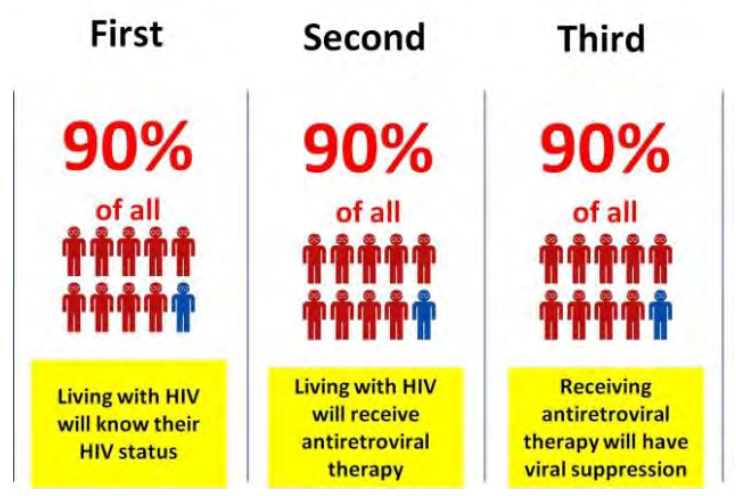

It urges to detect $90 \%$ from the invisible infected people in the geographical area concerned and treat $90 \%$ of identified, followed by adequate suppression of HIV viraemia among $90 \%$ of those on treatment.

But, triple-90 has no emphasis on behavioural determinants of HIV infection and generation of new infections. Its slogan is to detect and treat. It is a treatment target to help in ending AIDS. Therefore, our prevention programmes across all populations including key populations and other measures need to be kept tight while trying to achieve triple-90.

\section{Global scenario in ending AIDS}

Now, we will see how the global scenario in ending AIDS
Globally HIV new infections per year reached its peak in 1997, at 3.3 million new infections (95\% uncertainty interval [UI] 3·1-3.4 million). Annual HIV new infections declined fast from 1997 to 2005. However, it stayed relatively constant at about 2.6 million per year (range 2.5-2.8 million) since 2005.

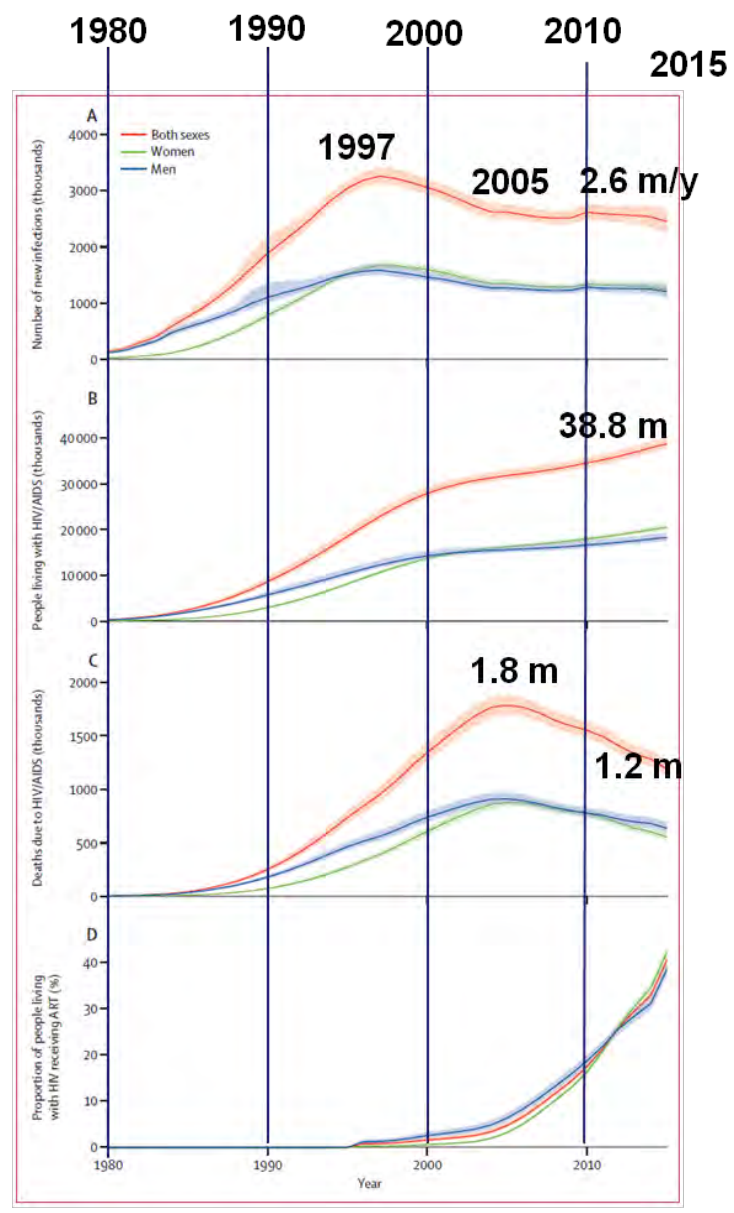

As mentioned in the Global Burden of Diseases Study 2015, published in Lancet, the number of people living with HIV/AIDS has been steadily increasing and reached 38.8 million (95\% UI 37.6-40.4 million) in 2015.

At the same time, HIV/AIDS mortality has been declining at a steady pace, from a peak of 1.8 million deaths ( $95 \%$ UI 1.7-1.9 million) in 2005 , to $1 \cdot 2$ million deaths ( $1 \cdot 1-1 \cdot 3$ million) in 2015 .

Scale-up of ART and prevention of mother-tochild transmission has been one of the greatest successes of global health in the past two decades. 
According to the study, of the 195 countries included in the study, 102 experienced an increase in the annual number of new HIV infections between 2005 and 2015. The study recorded a substantial heterogeneity in the levels and trends of HIV/AIDS across countries- Although many countries have experienced decreases in HIV/AIDS mortality and in annual new infections, other countries have had slowdowns or increases in rates of change in annual new infections.

The bitter truth is that no country has yet achieved the 90-90-90 target.

Globally only $41 \%$ of people living with HIV are receiving antiretroviral therapy (ART), and coverage of treatment remains low in many healthcare settings.

After three decades of interventions against HIV, still over 2 million new HIV infections occur annually. Whether we like it or not, it shows a failure; a failure by all stakeholders.

What do you think? Can we end AIDS by 2030, globally?

Is it

Possible?

Plausible?

Or a distant prospect?

I know, predicting uncertainties are difficult, especially when you do not have knowledge on potential predictors.

I think, ending AIDS globally is a distant prospect, unless all countries intensify their efforts.

\section{HIV epidemic overview in Sri Lanka}

As you all know Sri Lanka is an Island country, with a population of about 21 million living in a land area of approximately 65,000 square kilometres. It is a multilingual, multiethnic and multilingual country.

In Sri Lanka, first case of HIV reported in 1987 and since then there has been a gradual increase in annual HIV case rate as reported to the National STD/AIDS Control Programme. As of end second quarter 2016, 2,436 cumulative number of cases reported in Sri Lanka. During the last three years annual case rate was around 230. However, five years back in 2010 it was 121 .

Currently, it is estimated that about 4,100 people are living with HIV and every year < 500 new infections are being added and about $<100$ number of estimated AIDS deaths occur. Adult HIV prevalence is $<0.1 \%$

No province or district is exempted of HIV. However, analysis of new cases reported in 2015 showed that most of them were reported in the districts of Colombo, Gampha, Puttalam, and Kurunegala. New case rate per 100,000 populations in Colombo, Gampaha and Puttlem districts was 2.6, 2.3 and 1.8 respectively. Further, new case rate for Vavuniya, Kegalle, Kurunegala and Anuradhapura were 1.7, 1.4, 1.2 and 1 respectively

Analysis of main mode of transmission in each year from 2003 to 2015 shows that the main mode of transmission is heterosexual and the heterosexual epidemic is more prominent. However, there is gradual increase in the proportion of homosexual transmissions over the last years. Perinatal transmission ranges from $2-8 \%$ during the period. No cases have been reported due to blood and blood product transfusions.

Distribution of HIV cases by the age shows that majority of cases are reported in the age group of 25-49 years. It is important to note that about 32 cases are reported in the age group of 15-24 years which is used as the proxy value for new HIV infections.

Sero-positivity rate of different sub populations gives an overall picture of the HIV situation in the country. Testing different population groups in non-probability samples in various settings is shown in the table. The sero-positivity as you see, ranges from $0.003 \%-0.24$. 
However, in recently concluded integrated biological and behavioural survey 2014/15 (IBBS), different subpopulations tested in probability samples and the sero-prevalence among Female Sex Workers was 0.81\%, MSM $0.88 \%$ However, FSWs and MSM prevalence in the districts of Colombo and Galle is $1 \%$ while injecting drug users and beach boys prevalence was $0 \%$.

Condoms as a prevention (CasP) tool has been socialized among high risk groups to some acceptable level, according to the IBBS survey. Ninety three percent (93\%) of female sex workers had used condoms with their last client. Condom use among MSM was $58 \%$ and the same figure for beach boys. Among people who inject drugs it was $68 \%$. According to the IBBS, condom use has reached to $60 \%$ or beyond but issue is that whether the coverage of these populations is enough because of its hidden nature of the behaviour.

\section{Ending AIDS in Sri Lanka}

Sri Lanka should follow the 90-90-90 triple target to end AIDS by 2030 or even before 2030 , or if possible need to go beyond $90 \%$.

Out of the triple-90, the first 90 is the most difficult target in Sri Lanka, we need to identify $90 \%$ of infected people in the entire population, which is an invisible population.

Estimate says, about 4100 people living with HIV at any given point. However, we could link to services nearly about 1000 PLHIV, that is about $25 \%$ of the estimate.

Therefore, about 3000 people are living with HIV, but missing faces in our treatment services.

First

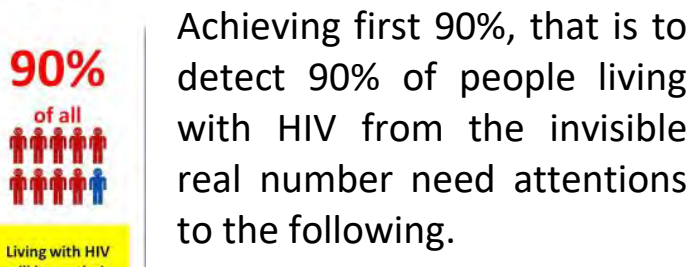

More robust data need to be fed for realistic estimation and projections to see whether we are working with close number to the actual invisible number.

Widespread testing for case detection is a must and need to take necessary steps to mainstream HIV testing to all possible entry points in health or non-health sectors. Key populations coverage is a priority but need to make it available freely beyond KPs for case detections.

It is very important to link all diagnosed people with HIV to HIV care services through follow up and support services.

Networking and active case finding is one of the cost effective and easiest way to detect positive cases.

Creation of enabling environment by removing or mitigating barriers for access for testing and care through legal and policy reforms and strengthening right based approaches.

Making structural changes in healthcare delivery, scale up Sexual Health services and ART centres to every corner of the country adhering to the principles of Universal Health Coverage (UHC).

Enabling environment at healthcare systems need to be established by reducing HIV stigma and discrimination ensuring confidentiality and making culturally competent workforce.

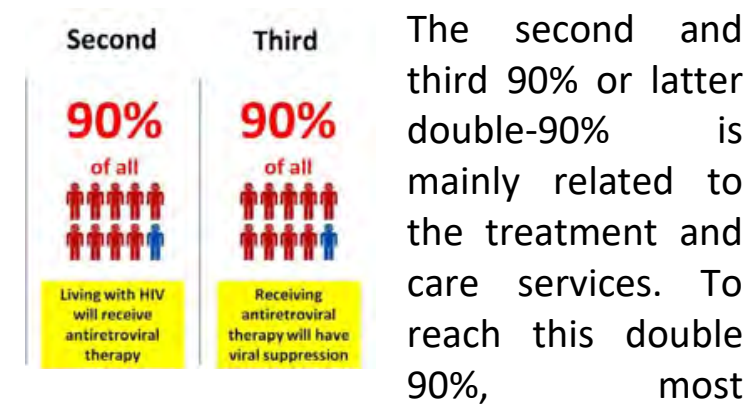

important factor is to retain PLHIV in care. 
Favourable legal and policy environment need to be in place to strengthen right to health and non-hesitant access to treatment and care.

Further, enabling social environment for PLHIV need to be created by removing or mitigating social barriers such as stigma and discrimination, marginalization, homophobia, transphobia, lack of acceptance of sexual diversity, denial attitudes of the society for sex and sexuality.

The supportive family environment is also important for PLHIV to retain in treatment and care.

We have paid less attention about personality issues and mental health which can reduce the retention in care such as anxiety, depression, non-concerned health behaviour, self stigma, religious fatalism, and personal attitudes towards treatment.

Enabling environment at healthcare systems need to be strengthened by training of staff to be culturally competent and sensitive. Low level of defaulter tracing and emphasis on partner servicers common due to conflicts of rights, but new strategies need to be implemented ensuring the rights of people.

Viral load testing need to be made available at an accessible and affordable distance.

Treatment adherence need to be strengthened.

Deviations for CAMs (complementary and alternative medicines) is another issue.
HIV clinicians and care service can play a major role in achieving the latter double- $90 \%$. I think Sri Lanka can achieve the latter double $90 \%$ quickly with the adaptation of "test and treat" policy and free and accessibility of Viral Load testing, HIV resistance testing and availability of treatment options.

Ending AIDS in Sri Lanka is not easy unless we intensify all our efforts. Therefore, all we need is to work towards ending AIDS in Sri Lanka before 2030 without making it a distant prospect.

There is no way of stepping back; we need to move forward and intensify prevention efforts. We need to scale up treatment and care services. We need to invest money for innovations. Finally most importantly make all possible efforts to deliver the evidence of science to our community.

Finally, I would like to recommend the ministry of health and the National STD/AIDS Control Programme, Sri Lanka to form a National steering committee and identify plan of action to make the ending AIDS a reality, which is possible.

Thank you

\section{Acknowledgement}

1. Dr Ariyaratne Manathunge, and the staff of the strategic information management unit of the National STD/AIDS Control Programme for generating important information

2. Dr Sisira Liyanage, Director, National STD/AIDS Control Programme

3. Office bearers and the Council of the college of Venerologists

4. Dr Sriyakanthi Beneragama for HIV and IBBS data 